\title{
Population dynamics of Vibrio spp. associated with marine sponge microcosms
}

\author{
Maria Hoffmann ${ }^{1,2}$, Markus Fischer ${ }^{2}$, Andrea Ottesen ${ }^{1}$, Peter J McCarthy ${ }^{3}$, Jose V Lopez ${ }^{4}$, \\ Eric W Brown ${ }^{1}$ and Steven R Monday ${ }^{1}$ \\ ${ }^{1}$ Division of Microbiology, Center for Food Safety and Applied Nutrition, US Food and Drug Administration, \\ Paint Branch Parkway, College Park, MD, USA; ${ }^{2}$ Department of Chemistry, Institute of Food Chemistry, \\ University of Hamburg, Grindelallee 117, Hamburg, Germany; ${ }^{3}$ Center for Marine Biomedical and \\ Biotechnology Research, Harbor Branch Oceanographic Institute at Florida Atlantic University, Fort Pierce, \\ FL, USA and ${ }^{4}$ Oceanographic Center, Nova Southeastern University, Dania Beach, FL, USA
}

\begin{abstract}
Vibrio is a diverse genus of marine-associated bacteria with at least 74 species and more expected as additional marine ecospheres are interrogated. This report describes a phylogenetic reconstruction of Vibrio isolates derived from one such unique ecosystem, marine sponges (Phylum Porifera) collected from depths of 150 to 1242 feet. 16S rRNA gene sequencing along with molecular typing of 16S-23S rRNA intergenic spacer regions clustered many sponge-associated Vibrio (spp) with current known species. That is, several benthic Vibrio species commensal with Porifera sponges seemed genetically linked to vibrios associated with coastal or shallow-water communities, signalling a panmictic population structure among seemingly ecologically disparate strains. Conversely, phylogenetic analysis provided evidence for at least two novel Vibrio speciation events within this specific sponge microcosm. Collectively, these findings earmark this still relatively unknown environment as a bastion of taxonomic and phylogenetic variability for the genus and probably other bacterial taxa.
\end{abstract}

The ISME Journal (2010) 4, 1608-1612; doi:10.1038/ismej.2010.85; published online 1 July 2010 Subject Category: microbial ecology and functional diversity of natural habitats

Keywords: 16S rRNA gene sequencing; 16S-23S rRNA intergenic spacer region; Phylum Porifera; speciation; Vibrio

\section{Introduction}

The genus Vibrio comprises a diverse bacterial group consisting of at least 74 distinct species, many having been recently identified in the last several years. Vibrios belong to the class Gammaproteobacteria and are gram-negative, usually motile rods. Generally, the organisms are halophilic, mesophilic and chemoorganotrophic in nature and have a facultatively fermentative metabolism (Thompson et al., 2004). Many of these species, such as $V$. cholerae, V. parahaemolyticus and $V$. vulnificus, induce severe human disease, including gastroenteritis, after ingestion of contaminated raw or undercooked seafood, and wound sepsis (Gulig et al., 2005; Saravanan et al., 2007; Su and Liu, 2007). Vibrios retain remarkable biodiversity, persisting in a variety of geographic locales and eukaryotic hosts, including corals, molluscs,

Correspondence: M Hoffmann, Division of Microbiology, US Food and Drug Administration, Mailstop HFS-711, 5100 Paint Branch Parkway, College Park, MD 20740, USA.

E-mail: maria.hoffman@fda.hhs.gov

Received 5 February 2010; revised 27 April 2010; accepted 19 May 2010; published online 1 July 2010 sponges and zooplankton (Thompson et al., 2004). Furthermore, environmental characteristics, such as water temperature and salinity, are also known to influence the diversity of Vibrio spp. in the environment (Beaz-Hidalgo et al., 2010).

Given the multitude of aquatic environments harboring vibrios, it seemed reasonable to hypothesize that additional Vibrio species might exist in remote undersea environments. One such environment-marine sponges-consists of numerous sponge species inhabiting different depths and regions of benthic seas. Sponges maintain diverse symbiotic microbe populations that differ in composition from microbial populations of surrounding ambient waters (Hentschel et al., 2006; Taylor et al., 2007; Webster and Blackall, 2009). Moreover, sponge anatomy allows persistence of distinct microbiomes in the same individual. One such microbiome, for example, is the mesohyl (extracellular matrix), which consists of regions with varying $\mathrm{O}_{2}$ concentrations (Hoffmann et al., 2005). In this case, highly selective conditions may encourage speciation as microbial populations adapt from oxygen rich to anoxic regions. In this study, we examine the phylogenetic diversity of vibrios associated with commensal sponge hosts in the Phylum Porifera. 


\section{Materials and methods}

Vibrio isolates were collected at depths between 150 and 1242 feet from several geographic locales and different sponges (Figure 1) and are maintained in the Harbor Branch Marine Microbial Culture Collection. Isolation of Vibrio spp. from sponges was carried out as previously described (Sfanos et al., 2005). Genomic DNA was isolated from pure Vibrio cultures using the ZR Fungal/Bacterial DNA kit (Zymo Research, Orange, CA, USA).

16S rRNA gene sequence analysis and 16S-23S rRNA intergenic spacer (IGS) analyses were performed, as described previously (Hoffmann et al., 2010). PCR primers and reaction conditions used for 16S rRNA amplification and 16S-23S rRNA IGS analyses are listed in Supplementary Material. IGS PCR amplicons were resolved by capillary gel electrophoresis using the Agilent BioAnalyzer 2100 and the Agilent DNA 7500 Assay Protocol (Agilent Technologies, Santa Clara, CA, USA). DNA purification, 16S rRNA gene sequence and 16S-23S rRNA IGS analyses were repeated three times to assure accuracy.

16S rRNA amplicons were sequenced by Amplicon Express (Pullman, WA, USA) and aligned with 16S rRNA gene sequences from the US Food and Drug Administration Vibrio type strain collection and Vibrio 16S rRNA gene sequences available at Genbank (http://www.ncbi.nlm.nih.gov). DNA sequences were aligned in ARB (http://www. arb-silva.de) against the SSU ARB-Silva bacteria collection hosted by the Max Planck Institute for Marine Microbiology in Bremen, Germany (Ludwig et al., 2004; Pruesse et al., 2007). Phylogenetic analyses were performed using PAUP* version 4.0b10 (Swofford, 2003) and the Genetic Algorithm for Rapid Likelihood Inference (GARLI) software (Zwickl, 2006). New 16S rRNA gene sequences from 23 Vibrio isolates are available in GenBank under accession numbers GU223581-GU223603.

\section{Results and discussion}

Analysis of 16S rRNA gene sequences allowed construction of a maximum parsimony tree (Figure 1), as well as a maximum likelihood tree (Supplementary Material, Supplementary Figure S1), revealing similarities to known Vibrio species for which benthic Vibrio isolates were most closely related. It was noteworthy that both analytical methods produced identical tree topologies with respect to nearest neighbors of the feral sponge isolates. Furthermore, our 16S-23S rRNA intergenic sequence typing protocol produced excellent results for sponge-associated isolates and showed several interesting genotypic differences between these isolates and their shallow-water counterparts.

Phylogenetic analysis revealed several notable findings regarding the diversity of spongeassociated vibrios. It is noteworthy that Vibrio isolates obtained from a Scleritoderma collected in Curacao (N376, N377, N382, N380, K350, K324 and K323) could not be differentiated by 16S rRNA gene sequencing indicating species homogeneity to $V$. natriegens and $V$. alginolyticus. IGS-patterns, however, suggested that these strains are more closely related to $V$. alginolyticus. In fact, these patterns were virtually identical, differing by a single 400-bp band absent in V. alginolyticus (Figure 2). Similarly, other Vibrio strains were either identical or nearly so to other known Vibrio species based on 16S rRNA gene sequence comparisons. For instance, strain J462 had a $16 \mathrm{~S}$ rRNA gene sequence identical to Aliivibrio fischeri, while both strains J231 and J555 shared $\sim 99.9 \%$ 16S rRNA gene sequence identity with $V$. mediterranei. In contrast to the Curacao strains, however, J462 generated an IGS-type pattern dissimilar to A. fischeri in actual number of bands generated. In addition, IGS-profiles generated for strains J231 and J555 differed substantially from patterns retained by their closest neighbors. Finally, strains N418 and N384 were identified as putatively novel Vibrio species-16S rRNA gene sequences showed significant divergence from known Vibrio species for both of these isolates (Figure 1). N384 was at least 19 steps (nucleotide substitutions) removed from $V$. brasiliensis, and N418, most closely related to $V$. ichthyoenteri and V. scophthalmi, was separated by combined branch lengths of 15 and 13 steps, respectively. These distances far exceed the 1\% 16S rRNA gene sequence divergence threshold as described by Acinas et al. (2004) and Harayama and Kasai, (2006) as the criterion for new and emerging species. As 16S rRNA gene sequence analysis represents a highly stable marker, divergence among spongeassociated vibrios and known reference sequences points to a distinct evolutionary separation explained, in part, by probable adaptation to a commensal existence in sponges.

16S rRNA gene sequences placed the majority of sponge-associated vibrios within the Harveyi clade (Vibrio core) (Sawabe et al., 2007), which includes $V$. parahaemolyticus, $V$. harveyii, $V$. alginolyticus, $V$. natriegens, V. rotiferanius and V. campbellii, albeit precise species determinations were largely intangible. Follow-up inspection of IGS genotypes revealed that one group of Curacao strains could be differentiated from other members of the Harveyi clade differing by only one band from $V$. alginolyticus. Given the hyper-variable evolutionary structure of 16S-23S rRNA intergenic spacers, however, this subtle difference belies significant genetic diversity between strains. Rather, it is plausible that these vibrios, all from sponge environments, share a highly homogeneous (that is, population level) phylogenetic relationship with their shallow-water counterparts. Given the notable genetic homogeneity among these geographically disparate strains, it is reasonable to postulate a panmictic population structure (that is, members of the population move 


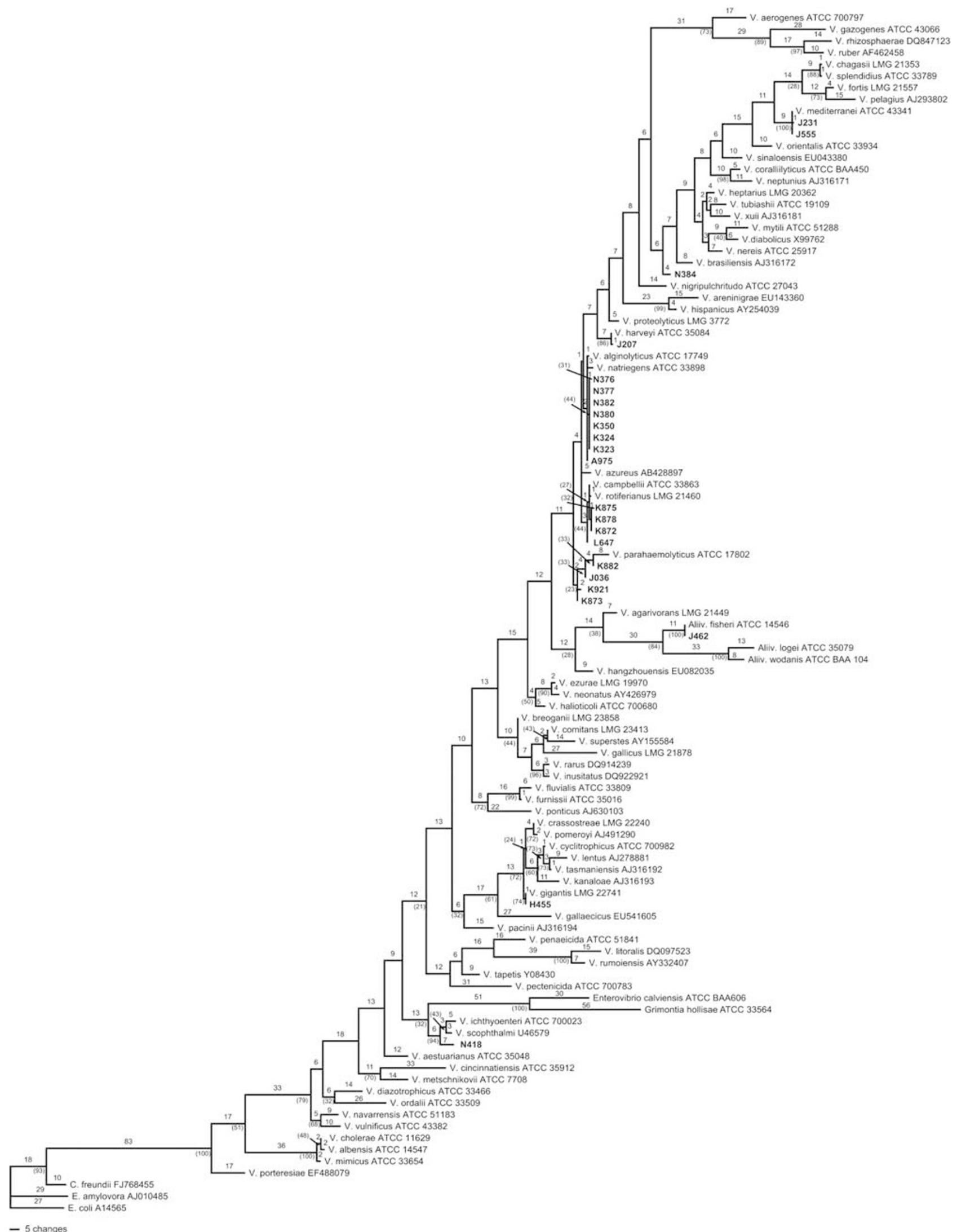


freely across habitats, likely are able to exchange DNA and share a common gene pool), whereby members freely associate in spite of the physical distances separating these two groups of vibrios. Although further studies are essential to understanding this relationship, other Vibrio hosts, such as zooplankton, for example, may facilitate transport between these two microbiomes.
Near-shore vibrios often associate in commensal relationships with oysters and other commercial shellfish species. Although these microcosms remain distinct from deep-water environs, the relationship between these ecologically disparate vibrios is important because novel species could be 'incidental' sponge residents as a consequence of filtration. It is, however, important to note that more

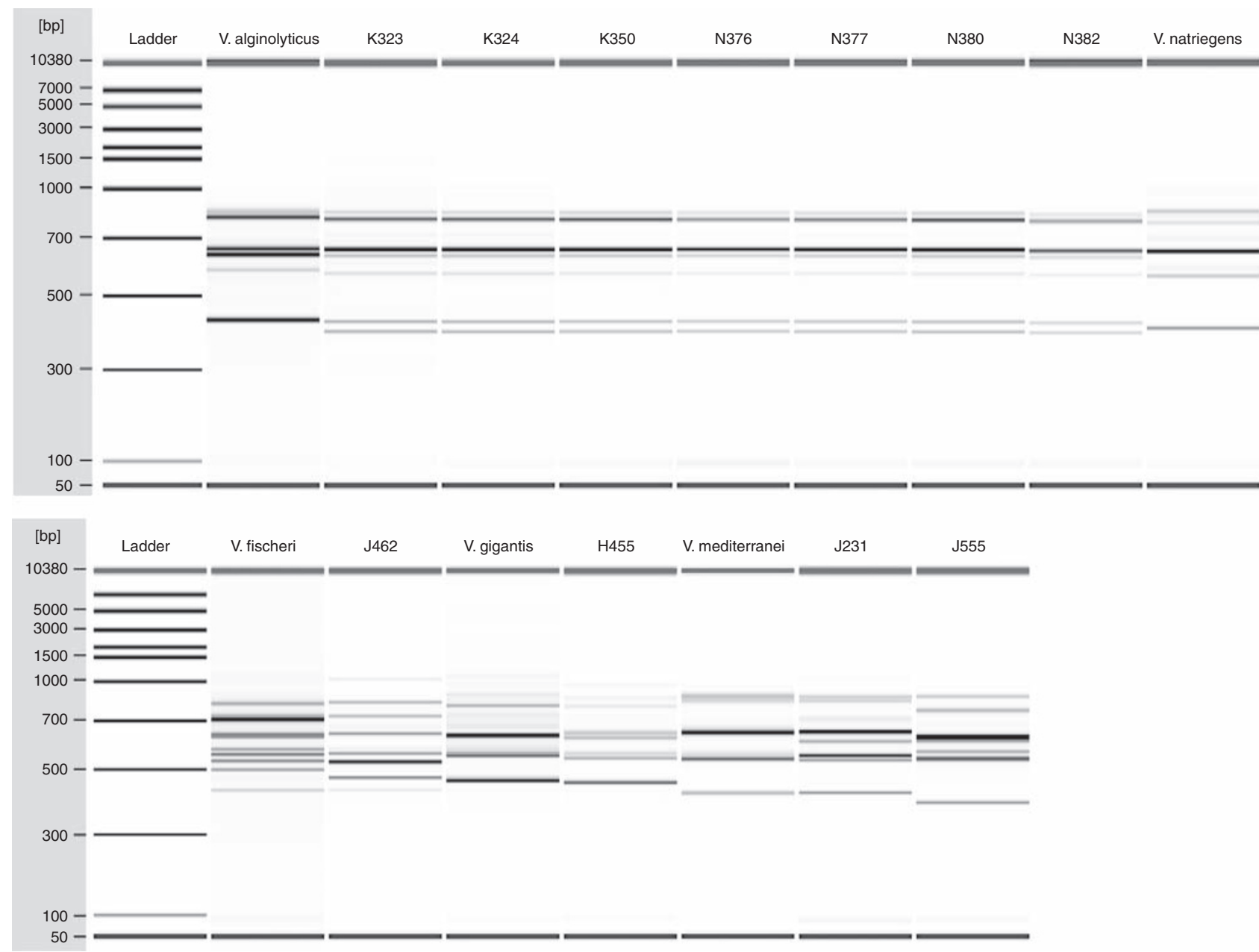

Figure 2 Representative virtual gel image, derived from the Agilent Bioanalyzer 2100, of 16S-23S rRNA IGS-type patterns of select Vibrio isolates and Vibrio reference species. Molecular weight standards are shown on the left of each gel image. Ln 1, Ladder; Ln 2, V. alginolyticus ATCC 17749; Ln 3, Vibrio K323; Ln 4, Vibrio K324; Ln 5, Vibrio K350; Ln 6, Vibrio N376; Ln 7, Vibrio N377; Ln 8, Vibrio N380; Ln 9, N382; Ln 10, V. natriegens ATCC 33898; Ln 11, Ladder; Ln 12, Aliivibrio fisheri ATCC 14546; Ln 13, Vibrio J462; Ln 14, V. gigantis LMG 22741; Ln 15, Vibrio H455; Ln 16, V. mediterranei ATCC 43341; Ln 17, Vibrio J231; Ln 18 , Vibrio J555.

Figure 1 Maximum parsimony (MP) tree derived from 16S rRNA gene sequences from 80 reference species and 23 Vibrio isolates. MP analysis was performed using PAUP* v.4.0b10 under heuristic search methods using tree bisection-reconnection (TBR), and 1000 random, stepwise addition replicates to generate the MP phylogram shown here. Bootstrap analyses were performed with identical parameters as used for the maximum parsimony analyses with 1000 replicates being considered. Values shown without parenthesis represent branch lengths, while those shown in parenthesis represent Bootstrap values. Bootstrap values $<20 \%$ were not shown. The tree was rooted with Escherichia coli, Erwinia amylovora, and Citrobacter freundii (GenBank accession numbers A14565, AJ010485 and FJ768455). Isolates K875, K882, K878, K921, K873 and K872 were cultured from Erylus sp. collected at 470 feet in the Bahamas; N376, N377, N382, N380, K350, K324, K323, N384 and N418 were cultured from Scleritoderma cyanea collected at 795 feet in Curacao; L647 was cultured from Corallistes sp. at 670 feet from Gulf of Mexico, FL, USA; J036 was cultured from Axinella sp. at 150 feet, H455 was cultured from Bubaris sp. at 260 feet and J207 was cultured from Higginsia strigilata at 200 feet from Atlantic seaboard, FL, USA; J231 and J555 were cultured from Halichondrida at 472 and 735 feet, respectively, and J462 was cultured from Pachastrellidae at 1242 feet from Puerto Rico. 
than 600 rRNA gene sequences taken from seawater showed little overlap with sequences derived from sponges (Hentschel et al., 2002). This supports the notion that sponge environments impose strong selection on resident microflora, generally prohibiting habitation by planktonic strains. Furthermore, these observations suggest that N384 and N418 were established commensal strains. At a minimum, some of these strains may qualify as 'ecotypes' in the sense of Cohan (Cohan, 2002), because sponge microcosms offer structural and ecological diversity that could propel dynamic bacterial speciation. Whatever their final evolutionary significance, it is clear that sponges represent a largely unexplored frontier harboring a virtual pantheon of genetic diversity for this important group of bacteria.

\section{Acknowledgements}

We would like to thank Marianna Naum for her assistance with the maximum likelihood analyses. This project was supported by an appointment of $\mathrm{MH}$ to the Research Fellowship Program for the Center for Food Safety and Applied Nutrition administered by the Oak Ridge Associated Universities. This work denotes HBOI contribution number 1805.

\section{References}

Acinas SG, Marcelino LA, Klepac-Ceraj V, Polz MF. (2004). Divergence and redundancy of $16 \mathrm{~S}$ rRNA sequences in genomes with multiple rrn operons. J Bacteriol 186: 2629-2635.

Beaz-Hidalgo R, Balboa S, Romalde JL, Figueras MJ. (2010). Diversity and pathogenecity of Vibrio species in cultured bivalve molluscs. Eviron Microbiol Reports 2: $34-43$.

Cohan FM. (2002). Sexual isolation and speciation in bacteria. Genetica 116: 359-370.

Gulig PA, Bourdage KL, Starks AM. (2005). Molecular pathogenesis of Vibrio vulnificus. J Microbiol 43: 118-131.

Harayama S, Kasai H. (2006). Bacterial phylogeny reconstruction from molecular sequences. In: E. Stackebrandt (ed.). Molecular Identification, Systematics and Population Structure of Prokaryotes. Springer: New York, pp 105-140.
Hentschel U, Hopke J, Horn M, Friedrich AB, Wagner M, Hacker J et al. (2002). Molecular evidence for a uniform microbial community in sponges from different oceans. Appl Environ Microbiol 68: 4431-4440.

Hentschel U, Usher KM, Taylor MW. (2006). Marine sponges as microbial fermenters. FEMS Microbiol Ecol 55: $167-177$.

Hoffmann F, Larsen O, Thiel V, Rapp HT, Pape T, Michaelis W et al. (2005). An anerobic world in sponges. Geomicrobiology J 22: 1-10.

Hoffmann M, Brown EW, Feng PCH, Keys CE, Fischer M, Monday SR. (2010). PCR-based method for targeting 16S-23S rRNA intergenic spacer regions among Vibrio species. BMC Microbiol 10: 90.

Ludwig W, Strunk O, Westram R, Richter L, Meier H, Yadhukumar et al. (2004). ARB: a software environment for sequence data. Nucleic Acids Res 32: 1363-1371.

Pruesse E, Quast C, Knittel K, Fuchs BM, Ludwig W, Peplies J et al. (2007). SILVA: a comprehensive online resource for quality checked and aligned ribosomal RNA sequence data compatible with ARB. Nucleic Acids Res 35: 7188-7196.

Saravanan V, Sanath Kumar H, Karunasagar I. (2007). Putative virulence genes of Vibrio cholerae from seafoods and the coastal environment of Southwest India. Int J Food Microbiol 119: 329-333.

Sawabe T, Kita-Tsukamoto K, Thompson FL. (2007). Inferring the evolutionary history of vibrios by means of multilocus sequence analysis. J Bacteriol 189: 7932-7936.

Sfanos K, Harmody D, Dang P, Ledger A, Pomponi S, McCarthy $\mathrm{P}$ et al. (2005). A molecular systematic survey of cultured microbial associates of deep-water marine invertebrates. Syst Appl Microbiol 28: 242-264.

$\mathrm{Su}$ YC, Liu C. (2007). Vibrio parahaemolyticus: a concern of seafood safety. Food Microbiol 24: 549-558.

Swofford DL. (2003). PAUP*: Phylogenetic Analysis Using Parsimony (* and Other Methods), version 4.0b 10. Sinauer Associates: Sunderland, MA.

Taylor MW, Radax R, Steger D, Wagner M. (2007). Spongeassociated microorganisms: evolution, ecology, and biotechnological potential. Microbiol Mol Biol Rev 71: 295-347.

Thompson FL, Iida T, Swings J. (2004). Biodiversity of vibrios. Microbiol Mol Biol Rev 68: 403-431.

Webster NS, Blackall LL. (2009). What do we really know about sponge-microbial symbioses? ISME J 3: 1-3.

Zwickl D. (2006). Genetic Algorithm Approaches for the Phylogenetic Analysis of Large Biological Sequence Dataset Under the Maximum Likelihood Criterion PhD thesis. University of Texas: Austin, TX.

Supplementary Information accompanies the paper on The ISME Journal website (http://www.nature.com/ismej) 